\title{
Intravitreal pegaptanib for the treatment of ischemic diabetic macular edema
}

\author{
This article was published in the following Dove Press journal: \\ Clinical Ophthalmology \\ II December 2015 \\ Number of times this article has been viewed
}

\section{Christine A Kiire \\ Rupal Morjaria \\ Anna Rudenko \\ Alexina Fantato \\ Lewis Smith \\ Amy Smith \\ Victor Chong}

Oxford Eye Hospital, Oxford

University Hospitals NHS Foundation

Trust, Oxford, UK
Correspondence: Victor Chong

Oxford Eye Hospital, Oxford University

Hospitals NHS Foundation Trust,

John Radcliffe Hospital, Headley Way,

Oxford OX3 9DU, UK

Email victor@eretina.com
Purpose: Pegaptanib has been shown to be effective in treating diabetic macular edema (DME) In the original Phase II/III trial, however, patients with macular ischemia were excluded. In this study, we treated patients with ischemic DME.

Methods: Macular ischemia was defined as a 30\% increase in the area of the foveal avascular zone (FAZ) at 45 seconds on fundus fluorescein angiography. In addition, the participants had diffuse foveal-involving DME with a central subfield thickness (CST) of $>300 \mu \mathrm{m}$ on spectral-domain optical coherence tomography. Five intravitreal pegaptanib injections were given 6 weeks apart. The final study visit was 6 weeks after the fifth injection. The primary outcome was change in the size of FAZ. Secondary outcomes were change in best-corrected visual acuity (BCVA) and the change in CST.

Results: Thirty participants were enrolled. Three were unable to complete the full course of treatment. Their outcomes were carried forward for the first part of this analysis. There was no statistically significant change in the mean size of the FAZ from baseline to the final visit. Subclassifying participants as those with minimal/moderate ischemia ( 16 participants, FAZ area $<1,000$ pixels) and those with more severe ischemia (14 participants, FAZ area $>1,000$ pixels) also showed no statistically significant change in the mean area of the FAZ. On average, BCVA increased and CST decreased from baseline to the final visit, but these changes were not statistically significant. Using per protocol analysis on those participants who completed the full course of treatment, the mean BCVA increased from 49.2 to 53.9 letters $(P=0.046)$.

Conclusion: In this study, intravitreal injection of pegaptanib did not significantly alter the size of the FAZ in participants with varying degrees of ischemic DME. There was, however, a significant improvement in mean BCVA in those who completed the treatment course.

Keywords: macular ischemia, anti-VEGF, pegaptanib, diabetic macular edema

\section{Introduction}

Diabetic macular edema (DME) is a leading cause of visual impairment in patients with diabetic retinopathy. ${ }^{1-3}$ A subset of these patients, those with macular ischemia, has a worse prognosis despite treatment with first-line approaches for DME such as focal/grid laser or intravitreal antivascular endothelial growth factor (anti-VEGF)-A. ${ }^{4}$ Standards for assessment of ischemic diabetic maculopathy were first established in the Early Treatment Diabetic Retinopathy Study (ETDRS) in the 1980s. ${ }^{5,6}$ Since then numerous studies have demonstrated a link between the presence of ischemic DME and the loss of visual function. ${ }^{5,7}$ The natural history and treatment benefits of ischemic DME remain unknown. ${ }^{5}$

Ranibizumab, a pan-VEGF-A blocker, was the first VEGF blocker approved for the treatment of DME. However, patients with ischemic DME were excluded in the pivotal study. ${ }^{8}$ Another pan-VEGF-A blocker, bevacizumab (Avastin), has also been 
reported to increase macular ischemia when used for the treatment of DME. ${ }^{9}$ Clinicians remain uncertain regarding the treatment options for patients with ischemic DME, with some studies suggesting that the presence of ischemia might lead to an adverse outcome with treatment or limit the benefits of treatment such as laser photocoagulation or intravitreal pharmacotherapies. ${ }^{5,10-12}$

Pegaptanib (Macugen, OSI/Eyetech, Melville, NY, USA) is a pegylated aptamer that targets the $\mathrm{VEGF}_{165}$ isoform, an especially proinflammatory isoform. ${ }^{13}$ It has been shown to inhibit the endothelial mitogen activity of VEGF-A and its vascular permeability effects. ${ }^{14}$ It is approved for the treatment of neovascular age-related macular degeneration. No systemic effects were attributed to the drug over the course of the study. ${ }^{13}$

A Phase II/III trial of pegaptanib for foveal-involving DME by the Macugen Diabetic Retinopathy Study group has shown benefit in eyes treated with intravitreal pegaptanib compared to sham-treated eyes. ${ }^{15}$ This study showed that the proportion of patients with $\geq 10$ letters of improvement in visual acuity at week 54 was statistically significantly greater in the pegaptanib versus the sham treatment arm. ${ }^{15} \mathrm{DME}$ with macular ischemia was not included in this study.

In our prospective, Phase IV, single-arm clinical trial, we have treated patients with varying degrees of macular ischemia, associated with DME, with intravitreal pegaptanib. We report the changes observed in the size of the foveal avascular zone (FAZ), best-corrected visual acuity (BCVA), and central subfield thickness (CST).

\section{Materials and methods}

Diabetic patients with a new diagnosis of DME with macular ischemia who had a BCVA of 20/32 to 20/320 were invited to join the study.

The participants received five injections of $0.3 \mathrm{mg}$ pegaptanib in the affected eye, 6 weeks apart. Changes in the size of the FAZ, CST, and BCVA were recorded over a 30 -week period. Patients with renal impairment (creatinine clearance $<20 \mathrm{~mL} / \mathrm{min}$ ) were excluded, based on the guidance from pegaptanib summary of product characteristics for age-related macular degeneration.
The primary objective of this study was to determine the effect of a series of 6-weekly injections of intravitreal pegaptanib on the size of the FAZ in ischemic DME over 30 weeks. Secondary objectives were to determine how a series of 6-weekly injections of intravitreal pegaptanib affects CST and BCVA in ischemic DME over 30 weeks (Table 1).

The primary end point was the change in the size of FAZ at 30 weeks, as measured in pixels using Adobe Photoshop. Secondary end points were the change in CST and BCVA at 30 weeks.

Trial participants were 30 consecutive, consenting patients with newly diagnosed ischemic DME. Inclusion and exclusion criteria are listed in Table 2. BCVA was recorded as number of letters +30 read on an ETDRS chart at $4 \mathrm{~m}$, or if no letters could be read at $4 \mathrm{~m}$, then the number of letters read at $1 \mathrm{~m}$. BCVA was recorded at baseline and then every 6 weeks for 30 weeks. A slit lamp examination was performed at each visit to look for any complications of the treatment. A spectral-domain optical coherence tomography (SD-OCT) scan was performed using Heidelberg Spectralis (Spectralis OCT, Heidelberg Engineering, software Version 1.6.4.0) to measure CST in microns at baseline and every 6 weeks. Fundus fluorescein angiography was performed using the Heidelberg Retina Angiograph II (HRA 2), at baseline and then at the third, fifth, and final visits to assess the size of the FAZ. The 45 second (or closest usable) fundus fluorescein angiography image (Figure 1) was used for the measurement of the FAZ. The image was exported to Adobe Photoshop for leveling to enable consistency between visits. The edges of the FAZ were demarcated and the area of ischemia was calculated in terms of number of pixels.

Intravitreal pegaptanib injections were performed in a clean room, under aseptic conditions, and according to the manufacturer's instructions. Participants were free to withdraw from the study at any time, for any reason, without prejudice to future care, and with no obligation to give the reason for withdrawal. In addition, the chief investigator had the right to discontinue treatment for a participant at any time if thought to be necessary for any reason, including pregnancy, ineligibility (either arising during the study or retrospectively, having been overlooked at screening),

Table I Objectives

\begin{tabular}{ll}
\hline Primary & Secondary \\
\hline To determine the effect of a series of five 6-weekly injections of & To determine how a series of five 6-weekly injections of intravitreal \\
intravitreal pegaptanib on the size of the FAZ in DME with macular & $\begin{array}{l}\text { pegaptanib affects central subfield thickness and BCVA in DME with } \\
\text { ischemia }\end{array}$ \\
\hline
\end{tabular}

Abbreviations: FAZ, foveal avascular zone; DME, diabetic macular edema; BCVA, best-corrected visual acuity. 
Table 2 Inclusion and exclusion criteria

\begin{tabular}{ll}
\hline Inclusion & Exclusion \\
\hline - Participant is willing and able to give informed consent for participation & - Any coexisting ocular disease (with the exception of cataract) \\
in the study & - Female participants who are pregnant, lactating, or planning \\
- Male or female, aged I8 years or above & pregnancy during the course of the study \\
- Participant has DME & - Any significant disease or disorder, for example, recent stroke or \\
- BCVA 20/32 to $20 / 320$ inclusive & myocardial infarction, which, in the opinion of the investigator, may \\
- Central OCT thickness $>300 \mu \mathrm{m}$ & either put the participants at risk because of participation in the \\
- Enlargement of FAZ (ischemia or capillary drop out of $>30 \%$ on FFA) & study, or may influence the result of the study, or the participant's \\
- Female participants of child bearing potential must be willing to ensure & ability to participate in the study \\
that they or their partner use effective contraception during the study & - Significant renal impairment, that is, creatinine clearance $<20$ mL/min \\
and for 3 months thereafter & - Participants who have participated in another research study \\
- Able (in the investigator's opinion) and willing to comply with all study & involving an investigational product in the past I2 weeks \\
requirements, for example, attending for tests and treatment every & - Within 3 months of laser treatment \\
6 weeks & - Intraocular surgery within 6 months \\
- Willing to allow his or her general practitioner and consultant, if & - Known allergy to pegaptanib \\
appropriate, to be notified of participation in the study & - Known allergy to fluorescein \\
\hline
\end{tabular}

Abbreviations: DME, diabetic macular edema; BCVA, best-corrected visual acuity; FAZ, foveal avascular zone; FFA, fundus fluorescein angiogram; OCT, optical coherence tomography.

significant protocol deviation, significant noncompliance with treatment regimen or study requirements, an adverse event that required discontinuation of the study medication or resulted in inability to continue to comply with study procedures, or disease progression which required discontinuation of the study medication (loss of vision of $>30$ letters). If a participant began the study with a visual acuity of $<30$ letters, then the study was discontinued if they could not see any letters. Under these circumstances, data were collected on affected participants, with their consent, for analysis on an "intention-to-treat" basis.

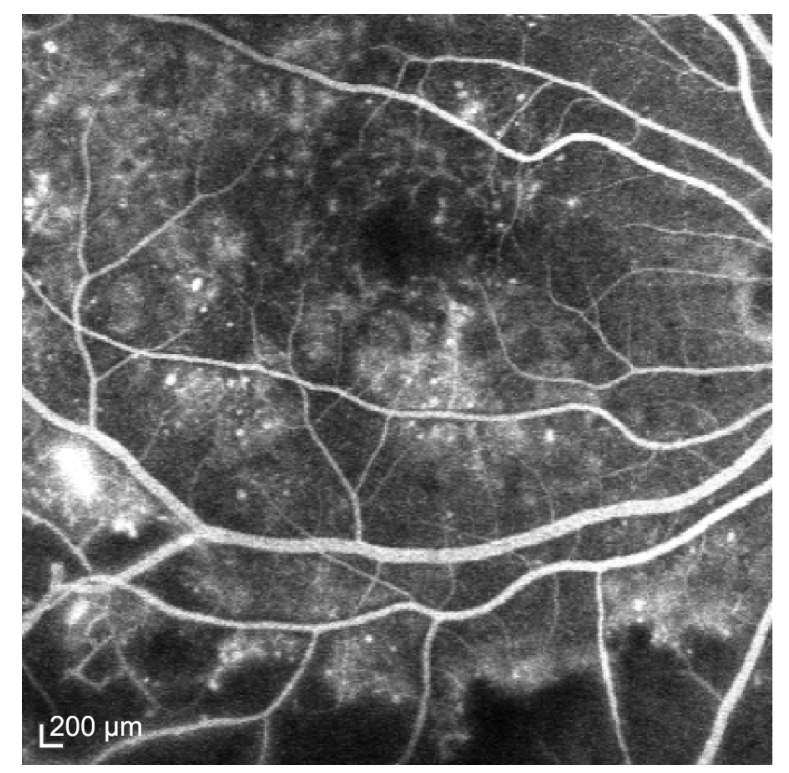

Figure I Fundus fluorescein angiogram at 45 seconds in a patient with ischemic diabetic macular edema.
Research funding was provided by Pfizer Ltd. However, Pfizer Ltd was not acting as sponsor for this study. It had no involvement in the design of the study protocol and analysis of the data. The study drug was provided by Pfizer Ltd, free of charge, for use in this study. It was stored and used according to the manufacturer's instructions. The treatment window was up to 3 weeks after the planned injection date. If an injection was given late, then the next injection was given no less than 4 weeks later. Participants were allowed to continue to receive treatment in the study if they missed one injection. After each pegaptanib injection the participant received a broad-spectrum antibiotic (ofloxacin $0.3 \%$ ) $4 \times / \mathrm{d}$ for 3 days. The final assessment was 6 weeks after the fifth intravitreal pegaptanib injection.

This study was conducted in accordance with the principles of the Declaration of Helsinki and in full conformity with the Data Protection Act and with the International Conference on Harmonisation Guidelines for Good Clinical Practice (CPMP/ICH/135/95) July 1996. Regular monitoring was performed by the University of Oxford Clinical Trials and Research Governance Unit. The protocol, informed consent form, and participant information sheet were approved by the South West London Research Ethics Committee 3 (REC reference 10/0803/58), the Medicines and Healthcare Products Regulatory Agency (EudraCT number 2009-017540-13), Oxford University Hospitals NHS Trust, and the University of Oxford Research and Development Department.

\section{Results}

There were 30 participants in this study. The mean age was 66.5 years (range $43-89$ years), and the male:female ratio was $2: 1$. Three patients were unable to complete the full course 
of treatment: one because of a drop in visual acuity of $>30$ letters, another because of being diagnosed with ischemic bowel while in the study, and another chose to discontinue treatment after three injections. The final results for these participants were carried forward for the purpose of the first part of this analysis.

The overall results of the study are summarized in Table 3. There was no statistically significant difference in the area of FAZ, CST, or BCVA from baseline to 30 weeks. Figures 2-4 show the change in FAZ area, CST, and BCVA, respectively, over the 30 -week study period.

If we analyze only those participants who completed the full course of treatment (per protocol analysis), there was a statistically significant increase in BCVA from 49.2 to 53.9 letters $(P=0.046)$ and close to significant reduction of CST from 478 to $424 \mu \mathrm{m}(P=0.052)$. There was no significant difference in the change in CST between those in the mild/ moderate ischemia group and those in the severe ischemia group $(P=0.844)$. Similarly, there was no significant difference in the change in BCVA between those in the mild/moderate ischemia group and those in the severe ischemia group $(P=0.0808)$.

Figure 5 shows an example of a patient who appeared to benefit significantly from treatment with pegaptanib. Having started with an acuity of 65 letters, FAZ area of 531 pixels and CST of $402 \mu \mathrm{m}$, the patient's BCVA improved by 12 letters to 77 letters. There was a slight reduction in the FAZ area to $476 \mu \mathrm{m}$ and a reduction in CST to $318 \mu \mathrm{m}$.

The serious adverse events we encountered are not believed to be related to pegaptanib and were discussed with our general medical physician colleagues. One participant was diagnosed with temporal arthritis and then 3 months later with gastroenteritis and cellulitis of the arm while undergoing treatment for gastroenteritis. The cellulitis may have been due to an infected intravenous cannula. Another participant developed cellulitis of the leg whilst undergoing study treatment. This participant, however, had a preexisting history of cellulitis. One patient developed ischemic bowel during the study, on a background of two episodes of unexplained suspected ischemic bowel that were treated on an inpatient basis with intravenous antibiotics and fluids a year prior to the commencement of the trial. Another participant lost 30 letters of vision despite two intravitreal pegaptanib injections. This patient had severe retinal ischemia. Another participant dropped out of the study because of difficulties attending the hospital every 6 weeks.

\section{Discussion}

The question of how to manage patients with signs of macular ischemia as well as DME is an important one. We add to the body of existing evidence with this study which shows that, in our cohort, pegaptanib did not alter the size of the FAZ significantly in patients with ischemic DME. Although there was a reduction in CST on OCT and an improvement in BCVA, this did not reach statistical significance, possibly due to the size of the study population. Only by analyzing the group who completed the full course of treatment did we see a statistically significant improvement in BCVA.

This is a small pilot study, without matched controls, but the implication of our findings is that it might be reasonable to give intravitreal pegaptanib to patients with DME, even if they are showing some signs of ischemic maculopathy. It is unclear whether pan-anti-VEGF-A drugs such as ranibizumab are safe in this group of patients. In our cohort, some patients had a dramatic improvement in their vision with this treatment. Overall there was a trend toward stabilizing vision with minimal change in the FAZ and the CST.

Table 3 Summary of results

\begin{tabular}{|c|c|c|c|c|c|c|}
\hline Patient group & $\begin{array}{l}\text { Number } \\
\text { of patients }\end{array}$ & $\begin{array}{l}\text { Mean age } \\
\text { (years) }\end{array}$ & $\begin{array}{l}\text { Outcome } \\
\text { measure }\end{array}$ & Baseline & End of study & $P$-value \\
\hline \multirow[t]{3}{*}{ Whole group } & 30 & 66.5 & FAZ (pixels) & 964 & 987 & 0.771 \\
\hline & & & $\mathrm{CST}(\mu \mathrm{m})$ & 491 & 451 & 0.128 \\
\hline & & & BCVA (letters) & 47.8 & 49.7 & 0.476 \\
\hline Mild/moderate ischemia & 16 & 68.9 & FAZ (pixels) & $44 I$ & 538 & 0.335 \\
\hline (FAZ area $<\mathrm{I}, 000$ & & & $\mathrm{CST}(\mu \mathrm{m})$ & 512 & 439 & 0.114 \\
\hline pixels) & & & BCVA (letters) & 45.3 & 47.8 & 0.587 \\
\hline Severe ischemia & 14 & 63.7 & FAZ (pixels) & I,562 & $\mathrm{I}, 500$ & 0.624 \\
\hline \multirow[t]{2}{*}{ (FAZ > I,000 pixels) } & & & $\mathrm{CST}(\mu \mathrm{m})$ & 467 & 466 & 0.938 \\
\hline & & & BCVA (letters) & 50.6 & 51.9 & 0.644 \\
\hline Those who completed & 27 & 67.0 & FAZ (pixels) & 973 & 946 & 0.734 \\
\hline the full course of & & & $\mathrm{CST}(\mu \mathrm{m})$ & 478 & 424 & 0.052 \\
\hline treatment & & & BCVA (letters) & 49.2 & 53.9 & 0.046 \\
\hline
\end{tabular}

Abbreviations: FAZ, foveal avascular zone; CST, central subfield thickness; BCVA, best-corrected visual acuity. 


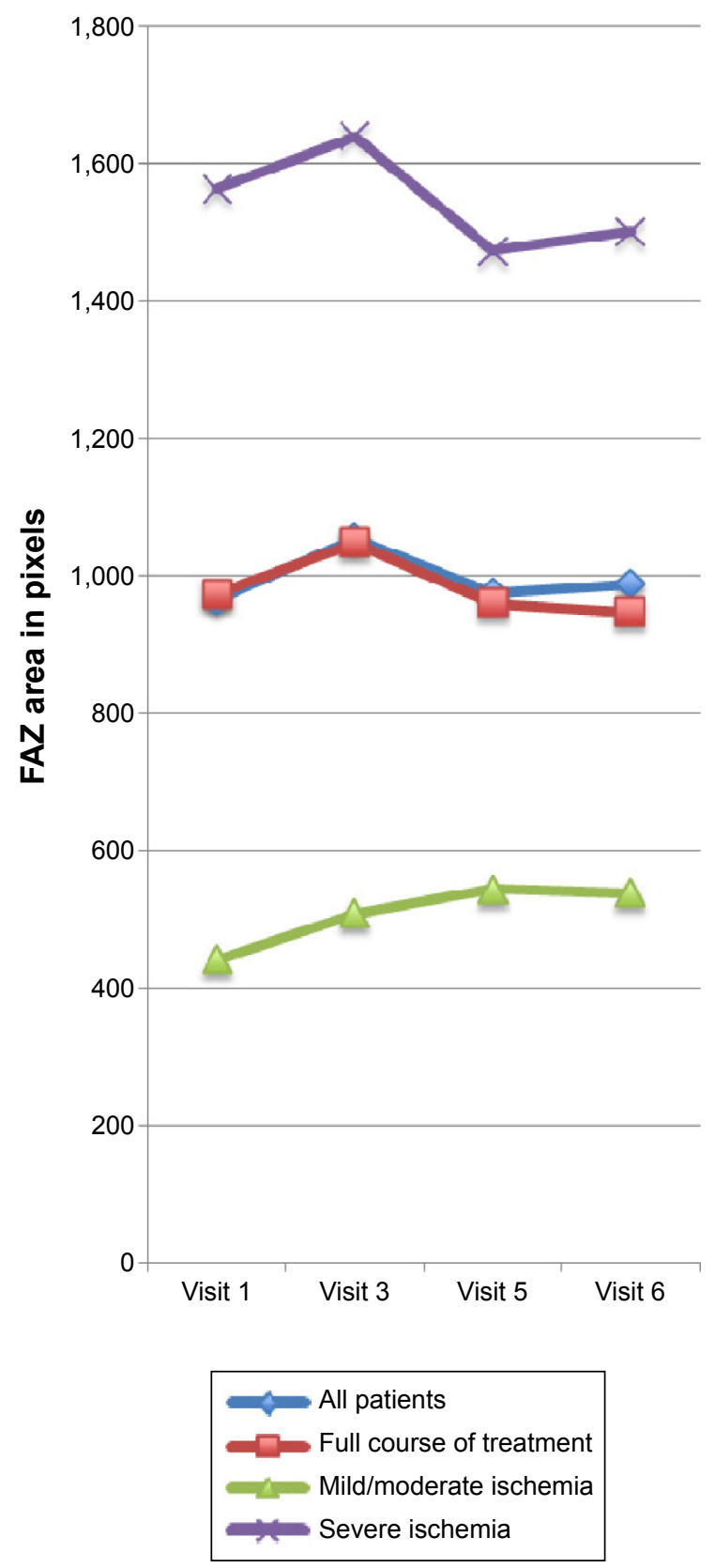

Figure 2 Mean change in FAZ area over 30 weeks.

Abbreviation: FAZ, foveal avascular zone.

The alternative to treating such patients is usually observation (without additional treatment) as they are not good candidates for laser, which has been shown to exacerbate ischemia. ${ }^{16}$ We have only used the selective VEGF-A antagonist, pegaptanib, but it might be worth investigating whether similar patients have the same results when all isoforms of VEGF-A are blocked. A comparison could be made between the outcomes of treated patients with those of untreated controls in order to establish the effectiveness of these drugs at stabilizing visual acuity in ischemic DME when compared to the natural history of the condition.

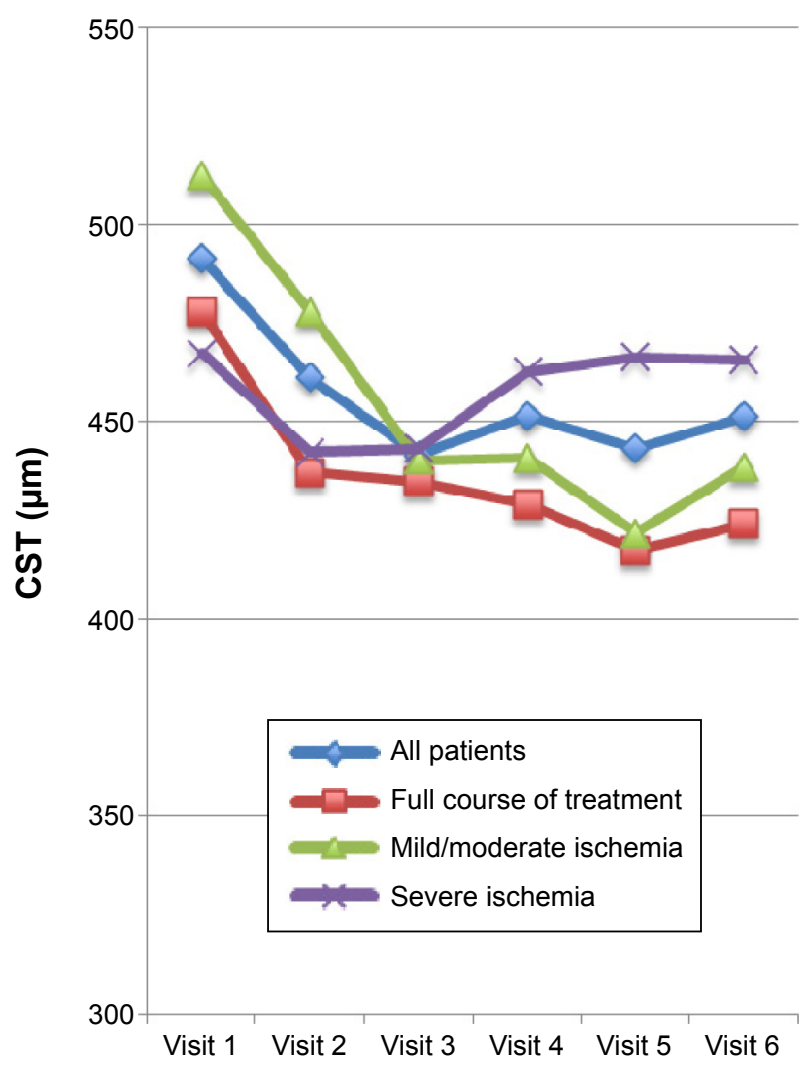

Figure 3 Mean change in CST over 30 weeks.

Abbreviation: CST, central subfield thickness.

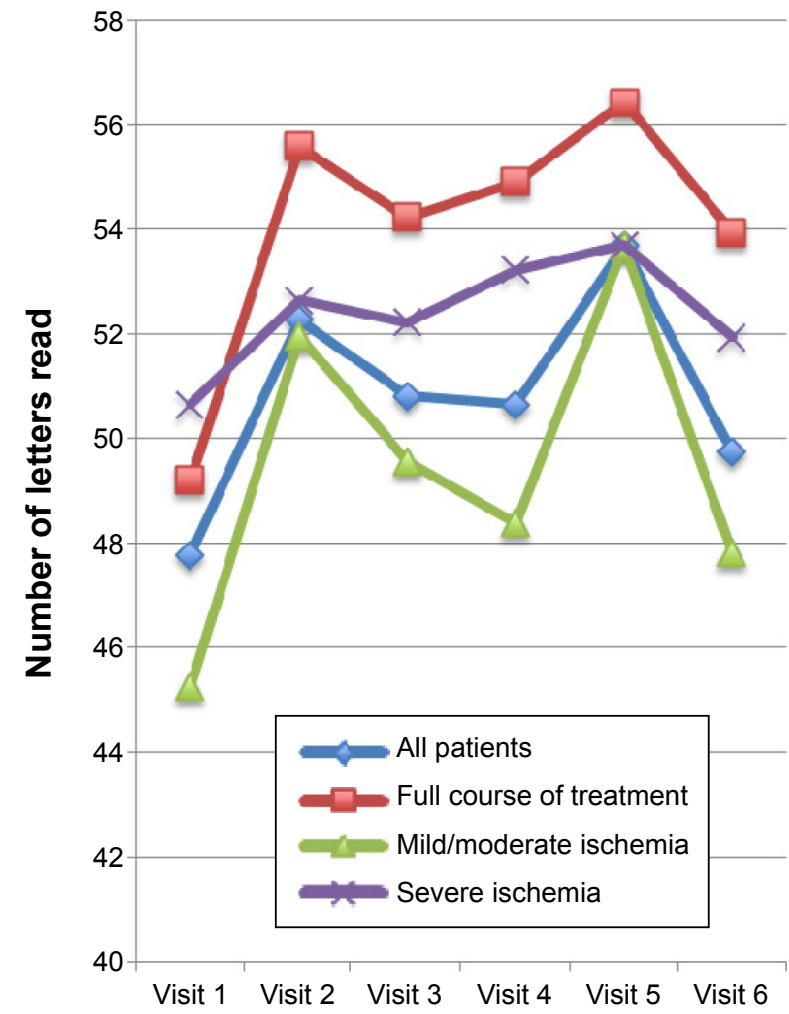

Figure 4 Change in BCVA over 30 weeks.

Abbreviation: BCVA, best-corrected visual acuity. 


\section{FAZ}

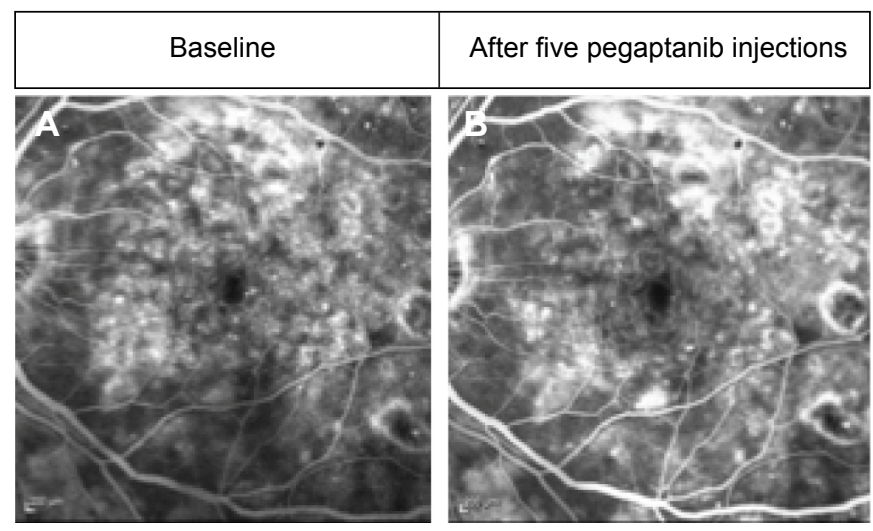

\section{OCT}

\section{BCVA}
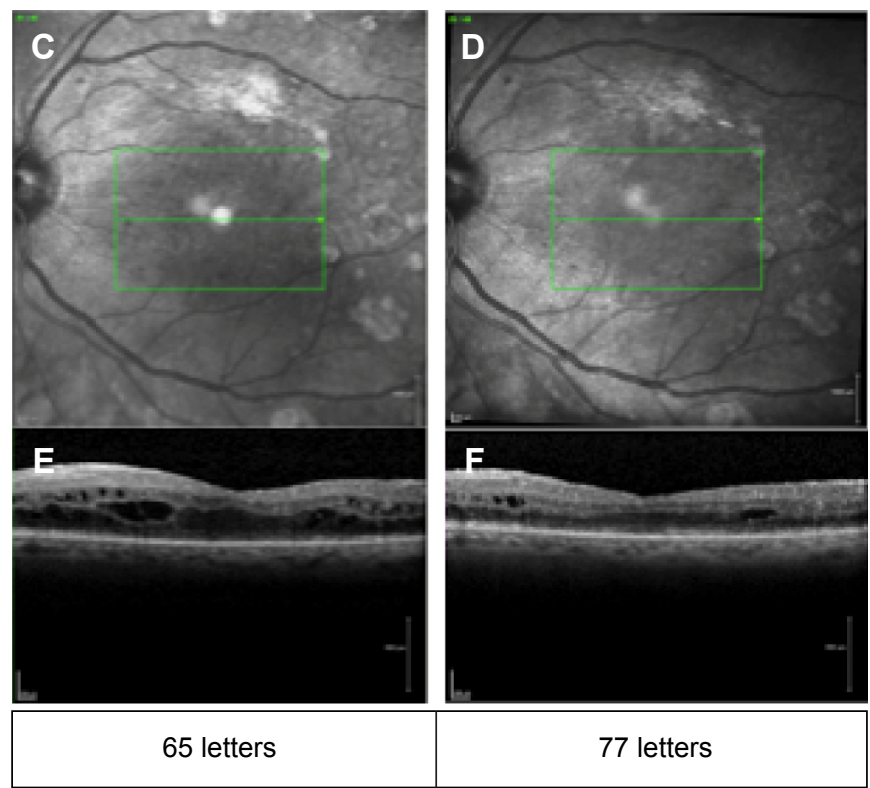

Figure 5 Example of a patient who benefitted from treatment.

Notes: Note the reduction in macular edema on OCT with minimal change in the size of the FAZ. (A) FAZ at baseline. (B) FAZ after five pegaptanib injections. (C and E) OCT scan at baseline. ( $\mathbf{D}$ and $\mathbf{F}$ ) OCT scan after 5 pegaptanib injections.

Abbreviations: FAZ, foveal avascular zone; OCT, optical coherence tomography; BCVA, best corrected visual acuity.

\section{Conclusion}

In our pilot study, intravitreal injection of pegaptanib did not significantly alter the size of the FAZ in participants with varying degrees of ischemia and DME. In addition, it did not lead to an overall statistically significant improvement in BCVA or CST. However, some patients showed significant benefit similar to that reported in the Phase II/III Macugen for DME study, and there was a statistically significant improvement in BCVA for those who completed the treatment course. These findings suggest that it might be reasonable to consider treating patients with ischemic DME with pegaptanib, despite the fact that it is not currently approved for the treatment of DME. For those who do not respond to pegaptanib, other anti-VEGF-A agents might be considered, bearing in mind that some of the anti-VEGF-A agents have approval for the treatment of DME. Our study population was, however, excluded from the pivotal studies; therefore, it remains unclear whether these agents are effective in this group of patients.

\section{Acknowledgments}

In addition to the named authors, the authors would like to acknowledge the participation of the following people who acted as study doctors, examining and treating patients in this study: Li Ping Chow, Emily Fletcher, Robert Purbrick, Sami Habal, and Spyridon Charisis. The authors would also like to acknowledge the other photographers who helped collect imaging for this study: Jon Brett and Matt Robertson. These data were presented as a poster at the Association for Research in Vision and Ophthalmology 2013 Annual Meeting (Seattle, WA, USA) in May 2013. 


\section{Disclosure}

CAK, RM, AR, AF, and LS report no conflicts of interest in relation to this work. AS has received travel grants from Novartis, Bayer, and Allergan. VC is a consultant for Allergan, Bayer, Novartis, and Quantel Medical. As stated earlier, this research was funded by Pfizer Ltd but the company was not acting as sponsor for this study and had no involvement in the design of the study protocol or analysis of the data. The study drug was provided by Pfizer Ltd, free of charge, for use in the study.

\section{References}

1. Moss SE, Klein R, Klein BE. Ten-year incidence of visual loss in a diabetic population. Ophthalmology. 1994;101(6):1061-1070.

2. Paulus YM, Gariano RF. Diabetic retinopathy: a growing concern in an aging population. Geriatrics. 2009;64(2):16-20.

3. Hirai FE, Knudtson MD, Klein BE, Klein R. Clinically significant macular edema and survival in type 1 and type 2 diabetes. Am J Ophthalmol. 2008;145(4):700-706

4. Parravano M, Menchini F, Virgili G. Antiangiogenic therapy with anti-vascular endothelial growth factor modalities for diabetic macular oedema. Cochrane Database Syst Rev. 2009;(4):CD007419.

5. Sim DA, Keane PA, Zarranz-Ventura J, et al. The effects of macular ischemia on visual acuity in diabetic retinopathy. Invest Ophthalmol Vis Sci. 2013;54(3):2353-2360.

6. Classification of diabetic retinopathy from fluorescein angiograms. ETDRS report number 11. Early Treatment Diabetic Retinopathy Study Research Group. Ophthalmology. 1991;98(5 suppl):807-822.
7. Arend O, Wolf S, Harris A, Reim M. The relationship of macular microcirculation to visual acuity in diabetic patients. Arch Ophthalmol. 1995;113(5):610-614.

8. Nguyen QD, Brown DM, Marcus DM, et al; RISE and RIDE Research Group. Ranibizumab for diabetic macular edema: results from 2 phase III randomized trials: RISE and RIDE. Ophthalmology. 2012;119(4): 789-801.

9. Nakamura Y, Takeda N, Tatsumi T, et al. [Macular ischemia following intravitreal bevacizumab therapy for diabetic macular edema]. Nippon Ganka Gakkai Zasshi. 2012;116(2):108-113. Japanese.

10. Ticho U, Patz A. The role of capillary perfusion in the management of diabetic macular edema. Am J Ophthalmol. 1973;76(6):880-886.

11. Jonas JB, Martus P, Degenring RF, Kreissig I, Akkoyun I. Predictive factors for visual acuity after intravitreal triamcinolone treatment for diabetic macular edema. Arch Ophthalmol. 2005;123(10):1338-1343.

12. Chung EJ, Roh MI, Kwon OW, Koh HJ. Effects of macular ischemia on the outcome of intravitreal bevacizumab therapy for diabetic macular edema. Retina. 2008;28(7):957-963.

13. Singerman LJ, Masonson H, Patel M, et al. Pegaptanib sodium for neovascular age-related macular degeneration: third-year safety results of the VEGF inhibition study in ocular neovascularisation (VISION) trial. Br J Ophthalmol. 2008;92(12):1606-1611.

14. Ng EW, Adamis AP. Anti-VEGF aptamer (pegaptanib) therapy for ocular vascular diseases. Ann N Y Acad Sci. 2006;1082:151-171.

15. Sultan MB, Zhou D, Loftus J, Dombi T, Ice KS. A phase 2/3, multicenter, randomized, double-masked, 2-year trial of pegaptanib sodium for the treatment of diabetic macular edema. Ophthalmology. 2011;118(6): 1107-1118.

16. Abhary S, Burdon KP, Gupta A, et al. Common sequence variation in the VEGFA gene predicts risk of diabetic retinopathy. Invest Ophthalmol Vis Sci. 2009;50(12):5552-5558.
Clinical Ophthalmology

\section{Publish your work in this journal}

Clinical Ophthalmology is an international, peer-reviewed journal covering all subspecialties within ophthalmology. Key topics include: Optometry; Visual science; Pharmacology and drug therapy in eye diseases; Basic Sciences; Primary and Secondary eye care; Patient Safety and Quality of Care Improvements. This journal is indexed on

Submit your manuscript here: http://www.dovepress.com/clinical-ophthalmology-journal

\section{Dovepress}

PubMed Central and CAS, and is the official journal of The Society of Clinical Ophthalmology (SCO). The manuscript management system is completely online and includes a very quick and fair peer-review system, which is all easy to use. Visit http://www.dovepress.com/ testimonials.php to read real quotes from published authors. 